\title{
Transient response in aqueous solution of an anions porous silicon based sensor
}

\author{
Arturo Ramirez-Porras ${ }^{1, ~}$, Natalia Murillo-Quiros ${ }^{2}$ \\ ${ }^{1}$ CICIMA and Escuela de Fisica, Universidad de Costa Rica, San Pedro 11501, Costa Rica \\ ${ }^{2}$ Escuela de Fisica, Instituto Tecnologico de Costa Rica, Cartago, Costa Rica
}

Email address:

aramirez@fisica.ucr.ac.cr (A. Ramirez-Porras),nmurillo@tec.ac.cr (N. Murillo-Quiros)

To cite this article:

Arturo Ramirez-Porras, Natalia Murillo-Quiros. Transient Response in Aqueous Solution of an Anions Porous Silicon Based Sensor. International Journal of Materials Science and Applications. Vol. 3, No. 5, 2014, pp. 200-204. doi: 10.11648/j.ijmsa.20140305.20

\begin{abstract}
A porous silicon based sensor has been developed to detect anions in a salt solution by the application of DC pulses on a Semiconductor/Electrolyte system. The sensor performance can be explained invoking a model where charge accumulation in the semiconductor surface states directly affects their geometric capacitances. By varying anions concentrations in salt solution, the results show a fairly constant value of substrate resistance, whereas variations in those geometric capacitances depend on the anion concentration. The constancy of substrate resistance and variability of geometric capacitance constitute key points for the development of an anion solution sensor.
\end{abstract}

Keywords: Porous Silicon, Chemical Sensors, Surface States

\section{Introduction}

Although porous silicon was studied firstly as a potential material for applications in the development of optoelectronic devices [1], the interest has shifted to many other fields, one of them being the use of this material as a sensing surface for detection of chemical species in gas [2] or liquid phase [3], or for biomedical applications [4],[5]. To accomplish this objective, this material must fulfill a series of protocols requiring specificity in the detection of particular molecules. One of such examples is the capability to sense pollutant salts in water, specifically anions [3]. One way to perform anion detection in such ambient is to use the Semiconductor/Electrolyte system [6], where an electrolyte containing anions is put in contact with the porous surface. By applying voltage pulses of a few volts to the system, one can extract useful information about the surface sensor states submitted to different aqueous ambients [7]. The purpose of this article is to provide insights in the development of this kind of new sensors developed in our laboratory.

\section{Experimental Setup}

\subsection{Electrochemical Process}

Commercially available Boron doped silicon wafers with $20-50 \Omega$-cm in resistivity, (100) crystallographic planes, and $300 \mu \mathrm{m}$ in thickness, were sputtered with aluminum in the non polished side to form an ohmic contact. Pieces of $1 \times 1 \mathrm{~cm}^{2}$ were cut from the wafer and put on the bottom part of a Teflon cell. The cell had a circular hole which exposed an area of $0.13 \mathrm{~cm}^{2}$ to the electroetching solution. This solution consisted of a mixture of HF, deionized water and ethanol in the proportion 1:5:2 per volume. Figure 1 shows the setup.

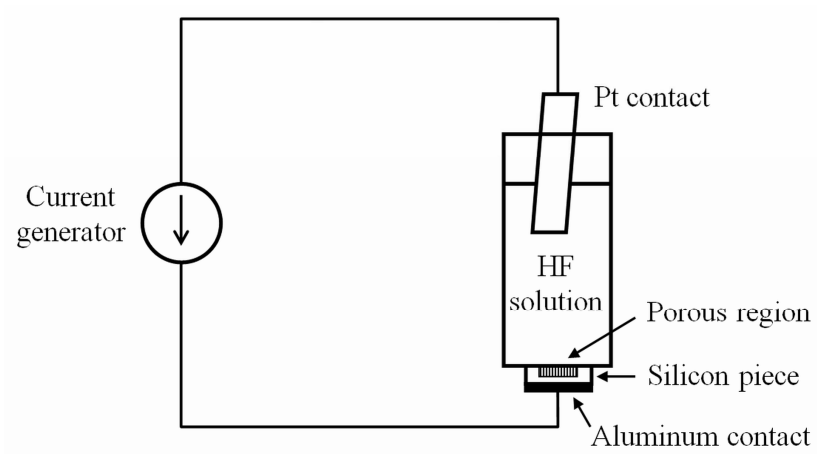

Figure 1. Experimental setup for porous silicon formation. A constant current source is directly connected to the back side of the Silicon piece. The circuit is closed with a contact made of platinum foil immersed in the etching solution. The porous region is formed on the upper side of the Silicon piece. 
A constant current density of $53 \mathrm{~mA} / \mathrm{cm}^{2}$ was applied across the system solution-wafer by a power generator (Agilent E3633A). A contact was placed at the bottom aluminum layer (anode) and the other was a platinum foil immersed in the etching solution (cathode). The etching time was set to 20 minutes. After the etching, the samples were rinsed with deionized water. Figure 2 show micrographs of the typical obtained layers.
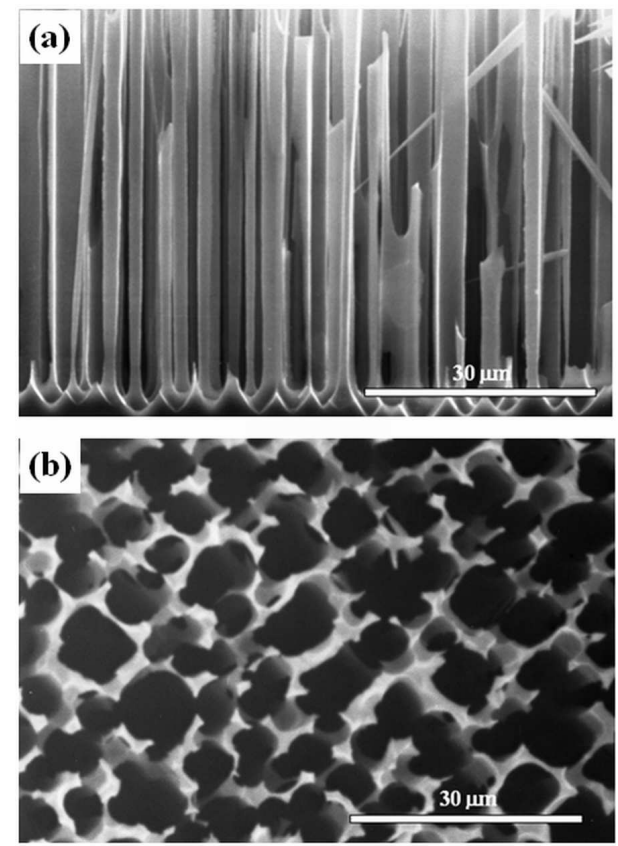

Figure 2. SEM photographs of typical porous layers. (a) Cross section, and (b) top view. The layer thickness was nearly $50 \mu \mathrm{m}$ and the pore diameters ranged in the 2-4 $\mu \mathrm{m}$. The scale of $30 \mu \mathrm{m}$ is shown in the photographs.

\subsection{Signal Measurement Process}

The samples were kept in the cell, the electrochemical solution discarded and replaced successively by three different test solutions of $\mathrm{KCl}$ : (a) $0.12 \mathrm{mmol} / \mathrm{l}$, (b) 0.23 $\mathrm{mmol} / \mathrm{l}$, and (c) $0.35 \mathrm{mmol} / \mathrm{l}$, corresponding respectively to $25 \mathrm{ppm}, 50 \mathrm{ppm}$ and $75 \mathrm{ppm}$ of $\mathrm{KCl}$ (mass per volume of water). The testing circuit is shown in Figure 3, where a pulse generator (Agilent 33220A) provided pulses of $10 \mu \mathrm{s}$ and heights between $400 \mathrm{mV}$ and $6 \mathrm{~V}$.

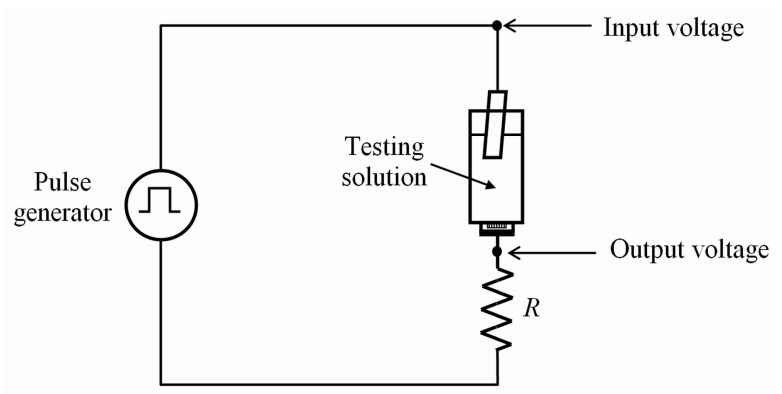

Figure 3. Experimental setup for sensor response to testing solutions. The input signal is provided by a pulse generator. The input and output voltages are determined by an oscilloscope. The resistor $R$ can be replaced by a capacitor $C$ whenever necessary.
The voltages were determined by the use of an oscilloscope (Tektronix TDS2012): one channel measuring the pulse provided by the generator ("input voltage") and the other channel measuring the voltage across a load resistor $R$ (as shown in Fig. 3) or a load capacitor $C$ (substituting $R$ in the circuit). All measurements were performed using the mentioned test solutions. We have shown previously [3] that this kind of sensor is able to detect anions in solution $\left(\mathrm{Cl}^{-}\right)$, so in this work no further mention of cations $\left(\mathrm{K}^{+}\right)$will be made.

\section{Modeling and Results}

\subsection{Circuit Model}

When a crystalline semiconductor is in contact with an electrolyte (the $S / E$ system), the charge transfer between both regions is essentially null. [6] Under the action of an external voltage pulse of a few microseconds width, changes in potential between the semiconductor and the electrolyte fall mostly across the space charge layer of the former (the layer that extends some microns just underneath the very first few atomic layers), and an equivalent geometric capacitance is able to precisely model the semiconductor space charge region. [8] Additionally, the charge that can be trapped on defects or dangling bond belonging to the actual surface layer can also be modeled by other geometric capacitance named the surface states capacitance. The full equivalent circuit is shown in Figure 4 [8].

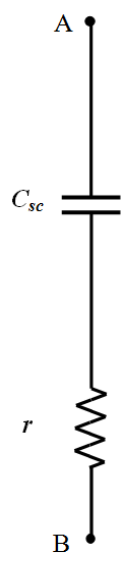

(a)

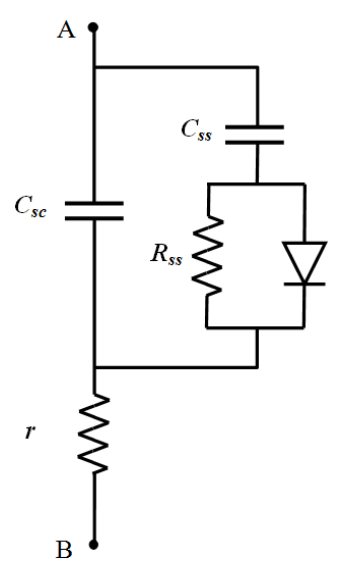

(b)
Figure 4. Equivalent circuits representing the $S / E$ system. (a) Perfectly blocking interface; (b) Presence of surface states.

In (a) is represented the simplest case where all charge in the semiconductor surface is stored in the space charge layer when the external pulse is applied. The resistor $r$ represents the equivalent resistance of the bulk semiconductor and the bulk solution. In (b), a network is placed in parallel to the space charge capacitance. This network is modeling the presence of surface states. The diode emphasizes the fact that the surface states capacitance can be charged almost instantly when the external pulse arises, but it takes a longer 
time to discharge (when the pulse sets off) due to the presence of a $R_{s S}$ resistor which models the high resistance values of the first surface layers.

Although this model was proposed for crystalline semiconductors, it has been successfully applied to porous silicon [7], [9]. The only observed difference is related to the higher area in contact with the electrolyte that does not affect the resistor-capacitor network model.

The methodology employed in this paper considers the response of the system to an external pulse. Figures 5 and 6 show the expected output voltage curves when the sensor model sketched in Figure 4(b) is used in series with an external resistor $R_{E}$ (Figure 5) or with an external capacitor $C_{E}$ (Figure 6). The inset plots depicts the expected behavior of the input and output signals (the voltage measured across the external element).
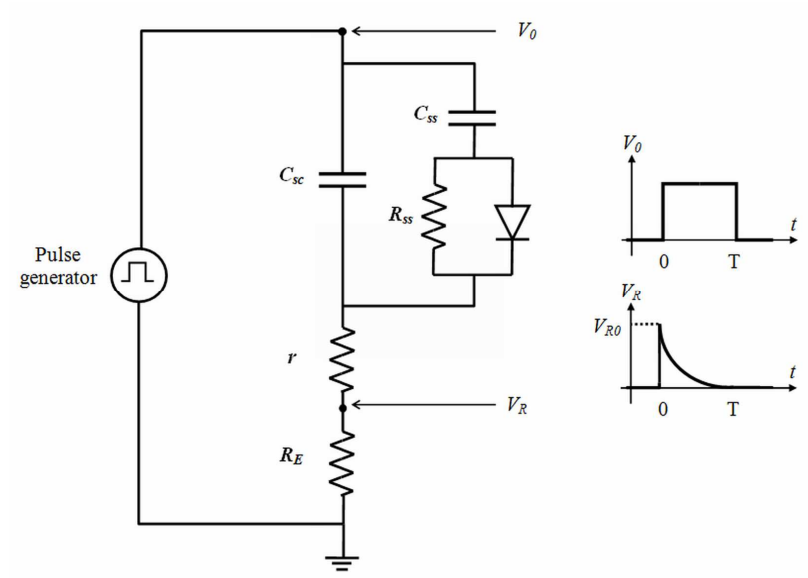

Figure 5. Model of the sensor using an external resistor in series. The inset plots show the input signal (upper) and output signal (lower).

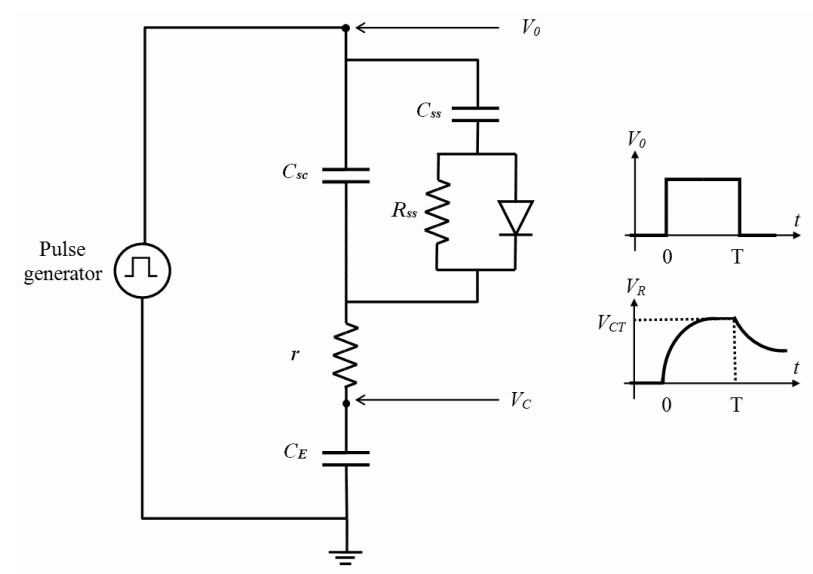

Figure 6. Model of the sensor using an external capacitor in series. The input and output signals are shown on the inset plots similarly to the case of Figure 5).

Consider the network with $R_{E}$ (Figure 5). When the pulse sets in, all capacitors are discharged and therefore act as shorts. As a consequence, the voltage across $R_{E}$ is a voltage divisor between $R_{E}$ and $r$. Its value can be calculated as:

$$
V_{R 0}=R_{E} V_{0} /\left(R_{E}+r\right)
$$

where $V_{0}$ is the height of the input pulse. Notice that $V_{R 0}$ is output voltage measured just on the onset of the pulse. On the other hand, if the pulse width $T$ is large enough, the capacitors would tend to charge until they reach a constant value and behave as open circuits. No more current flows then across $R_{E}$. Consequently, the voltage tends to zero near the end of the pulse. If $R_{E}$ is now replaced by $C_{E}$, on the onset of the pulse all capacitors are shorts and the output voltage is null. As the pulse progress to $T$, the capacitors charge up to their full value, where they open the circuit. Therefore, the output voltage at time $T$ is:

$$
V_{C T}=Q_{E} / C_{E}
$$

where $Q_{E}$ is the charge contained in the external capacitor. Note that, because no more charge flows in the circuit and $C_{E}$ is in series with $C_{S C}$ and $C_{S S}$ (which in turn are in parallel) the total charge $Q_{E}$ is the same as the sum of the charge contained in $C_{S C}$ and $C_{S S}$. Using this and the fact that: $V_{0}=Q_{E} / C_{E}+Q_{E} / C_{T O T}$, with $C_{T O T}=C_{S C}+C_{S S}$, it is straightforward to show that

$$
V_{C T}=C_{T O T} V_{0} /\left(C_{T O T}+C_{E}\right)
$$

The interest here is to find the unknown parameters, $r$ and $C_{T O T}$. Note that it is not possible to separate $C_{s c}$ and $C_{s s}$ from $C_{T O T}$. Nevertheless, if any change in charge arises for the different testing solutions, it can be attributed in principle to a change in the surface potential, and therefore this affects the surface states capacitance $C_{s s}$, consequently affecting the total capacitance as well.

Let the adimensional parameters $\alpha_{0}$ and $\beta_{T}$ be defined as:

$$
\alpha_{0}=V_{R 0} / V_{0}, \quad \beta_{T}=V_{C T} / V_{0}
$$

Inserting (4) into (1) and (3) and solving for $r$ and $C_{T O T}$, the results are:

$$
r=\left(\alpha_{0}^{-1}-1\right) R_{E}, C_{T O T}=\left(\beta_{T}^{-1}-1\right)^{-1} C_{E}
$$

With these expressions and the experimental determination of voltages, the internal resistance and capacitance can be obtained and correlated with the concentrations of the studied anion in the solution.

\subsection{Results and Discussion}

Figure 7 shows a plot of the internal resistance as a function of the input voltage for the three considered concentrations. The value of $R_{E}$ was $11.3 \mathrm{k} \Omega$. The general trend is that $r$ has a fairly constant value of $57 \mathrm{k} \Omega$ for all concentrations, independently of the input voltage. This result is expected because $r$ models the resistance of bulk semiconductor and solution. Although the solution conductivity changes with the presence of the added anions, this change is not strong enough to affect the overall bulk resistance. This could also indicate that the space charge layer of the semiconductor is not affected by the variations taking place at the very surface. Therefore, a DC pulse methodology for the determination of changes in bulk resistances for different chemical ambients is not useful for 
sensing purposes.

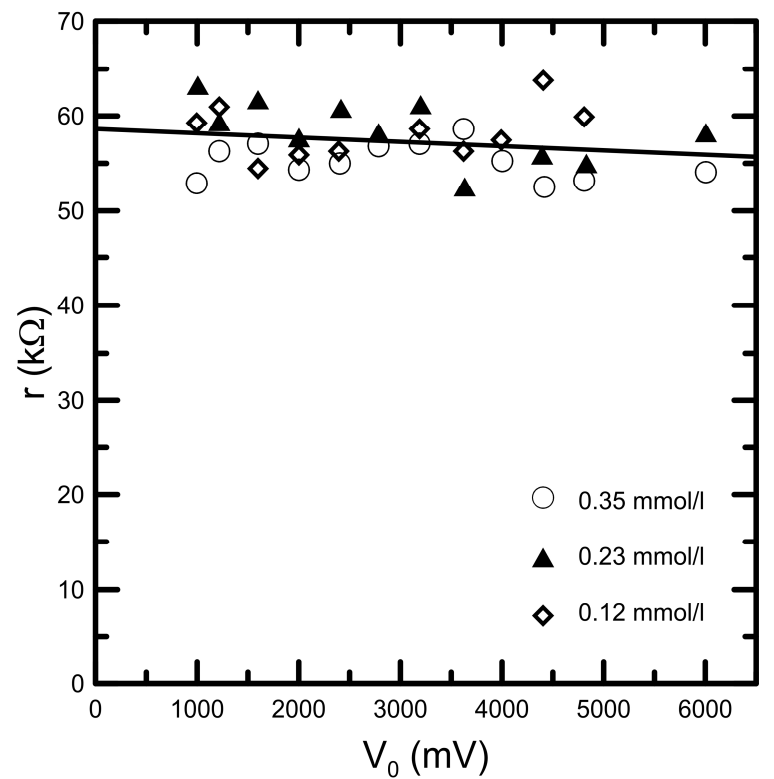

Figure 7. Internal resistance vs input voltages for all concentrations of $\mathrm{KCl}$, as marked.

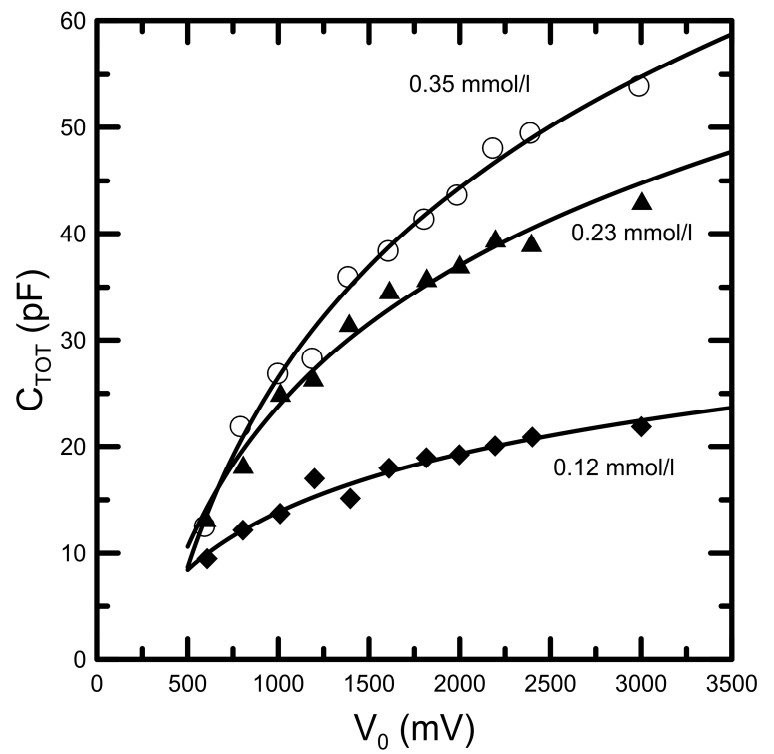

Figure 8. Internal capacitance plots $v$ s input voltages for the three different considered concentrations of $\mathrm{KCl}$, as marked..

Figure 8 shows the obtained internal capacitance plots in the three cases. The curves seem to be grouped at low voltages, but separate at higher values, showing a direct possibility to detect those small amounts. With respect to the resulting curve for $0.12 \mathrm{mmol} / \mathrm{l}$, the $0.23 \mathrm{mmol} / 1$ is two folded at an input voltage of $3 \mathrm{~V}$, and the $0.35 \mathrm{mmol} / \mathrm{l}$ is almost three folded. The strength of the input voltage for fair detectability lies in the 2 to 3 Volts range, normally used in the electronics industry. In our model, an increase in total geometric capacitance is attributed to an increase in the surface states geometric capacitance. The reason is the constancy of the internal resistance addressed above: the space charge layer of bulk semiconductor is unaffected and so is the space charge capacitance. This means that more charge is accumulated on the surface due to the presence of anions. Determination of geometric capacitances could be used therefore as a sensing parameter for anions detection.

\section{Conclusions}

A porous silicon sensor to detect anions in a salt solution has been presented. The sensor works using DC pulse regime and shows weak changes in internal resistance but strong variations in total geometrical capacitances when subject to various concentrations of the same salt. As a consequence, discrimination among different signals is possible to perform at least for concentrations between 0.1 and $0.4 \mathrm{mmol} / \mathrm{l}$. These variations are attributed to differences in the surface electronic states and not to the space charge layer of the semiconductor.

\section{Acknowledgments}

The authors acknowledge the financial support of the Vicerrectoría de Investigación of the Universidad de Costa Rica.

\section{References}

[1] O. Bisi, S. Ossicini, and L. Pavesi, "Porous silicon: a quantum sponge structure for silicon based optoelectronics," Surf. Sci. Rep., vol. 38, no. 1-3, pp. 1-126 ( 2000).

[2] G. Korotcenkov, Handbook of Gas Sensor Materials. Springer New York, 2013.

[3] N. Murillo, E. Rucavado, and A. Ramírez-Porras, "Effect of ethanol in an inorganic salt detector based on porous silicon," Phys. Status Solidi C, vol. 6, no. 7, pp. 1709-1712 (2009).

[4] T. R. Dargaville, B. L. Farrugia, J. A. Broadbent, S. Pace, Z. Upton, and N. H. Voelcker, "Sensors and imaging for wound healing: A review," Biosens. Bioelectron., vol. 41, pp. 30-42 (2013).

[5] S. Dhanekar and S. Jain, "Porous silicon biosensor: Current status," Biosens. Bioelectron., vol. 41, pp. 54-64, (2013).

[6] A. Many, N. B. Grover, and Goldstein, Semiconductor Surfaces, By A. Many, Y. Goldstein, and N.B. Grover. Amsterdam, North-Holland Pub. Co., 1965.

[7] S. Z. Weisz, A. R. Porras, M. Gomez, A. Many, Y. Goldstein, and E. Savir, "Relation between luminescence and electronic surface characteristics in p-type porous silicon," J. Lumin., vol. 72-74, pp. 729-730 (1997).

[8] M. Wolovelsky, J. Levy, A. Many, S. Z. Weisz, and O. Resto, "Space charge and surface state characteristics of the silicon/electrolyte interface," Surf. Sci., vol. 171, pp. 442-464 (1986). 
[9] A. Ramirez Porras, O. Resto, S. Z. Weisz, Y. Goldstein, A. Many, and E. Savir, "Luminescence and Surface-State Characteristics in P-Type Porous Silicon," MRS Online Proc. Libr., vol. 452 (1996). 